\title{
Partnering dispersion corrections with modern parameter-free double-hybrid density functionals
}

\author{
J. C. Sancho-García ${ }^{1 *}$ E. Brémond ${ }^{2 \dagger}$ \\ M. Savarese ${ }^{2}$, A. J. Pérez-Jiménez ${ }^{1}$, and C. Adamo ${ }^{2,3}$ \\ ${ }^{1}$ Departamento de Química Física, \\ Universidad de Alicante, E-03080 Alicante, Spain \\ ${ }^{2}$ CompuNet, Istituto Italiano di Tecnologia, \\ via Morego 30, I-16163 Genoa, Italy \\ ${ }^{3}$ Institut de Recherche de Chimie Paris, \\ IRCP CNRS UMR-8247, Chimie ParisTech, \\ École Nationale Superieure de Chimie de Paris, \\ 11 rue P. et M. Curie, F-75231 Paris Cedex 05, France
}

February 21, 2017

${ }^{*}$ E-mail: jc.sancho@ua.es

${ }^{\dagger}$ E-mail: Eric.Bremond@iit.it 


\begin{abstract}
The PBE-QIDH and SOS1-PBE-QIDH double-hybrid density functionals are merged with a pair of dispersion corrections, namely the pairwise additive D3(BJ) and the non-local correlation functional VV10, leading to the corresponding dispersion-corrected models. The parameters adjusting each of the dispersion corrections to the functionals are obtained by fitting to well-established energy datasets (e.g. S130) used as benchmark, giving rise to functionals spanning covalent and noncovalent binding forces. The application of the models to challenging systems out of the training set, like those comprising the L7 database of large supramolecular complexes, or the S66x8 dataset of stretched and elongated intermolecular distances, reveals the high accuracy of the coupling.
\end{abstract}

Key words: double-hybrid density functionals, dispersion corrections, S130, L7, and S66x8 datasets. 


\section{Introduction}

Intermolecular dispersion interactions are a major driving force for the structure, stability, and response properties of molecular complexes. As a matter of fact, they influence the formation of biologically active compounds [1], the adsorption processes in layered materials [2-4], the drug delivery [5], or the supramolecular chemistry [6], to name just a few examples. Consider an idealized case of two individual molecules, $M$ and $N$ each one bearing its own ground-state electronic distribution, which is averaged over a period of time large enough compared to the allowed fluctuations of the electron density so that the two elements are originally non-polar in nature. However, when they start to approach and thus to weakly interact, and if some asymmetry arises in the electron distribution of one of these elements, say $M$, a short-lived dipole moment will be consequently induced. If the element $N$ is also polarizable, the field arising from that created dipole will instantaneously influence the neighbouring elements, creating concomitantly a manifold of induced dipoles (or, in general, multipoles) up to a certain cutoff. The original dipole will necessarily change its value and orientation as a consequence of this field embedding, and then the induced dipoles will follow it, meaning that the large number of transient dipoles will not longer vanish, giving thus rise to an attractive potential (induced dipole - induced dipole) energy commonly known as London dispersion energy.

Thus, dispersion becomes the strongest component of non-covalent interactions in non-polar molecules; note that this physics picture also holds if one or both molecules are polar originally, although the non-covalent effects would now incorporate other electrostatic-driven contributions (i.e. Debye and Keesom dispersion forces, respectively) for this case like it happens for $\mathrm{H}-$ 
bond interactions. Therefore, dispersion interactions wholly originate from electron correlation effects, with mean-field approximations (i.e. HartreeFock theory) failing completely to capture them, and needing thus to be incorporated from more advanced (first-principles) theories such as ab initio or Density Functional Theory (DFT). However, in the case of the latter, the correlation functionals generally employed are local, that is, depending only on the density at some point $\mathbf{r}$ in space, $E_{c}[\rho(\mathbf{r})]$, or semi-local, when the gradient $\nabla \rho(\mathbf{r})$ and eventually some other higher-order derivatives are also incorporated into the functional, $E_{c}[\rho(\mathbf{r}), \nabla \rho(\mathbf{r})]$, missing thus the longrange correlation acting between electrons situated at different points $\mathbf{r}$ and $\mathbf{r}^{\prime}$ in space. Despite it, and fortunately, dispersion-corrected theories have currently become easily available (for some reviews see Refs. [7-18]) to overcome this situation and thus to properly describe the phenomena of molecular complexation when DFT is employed.

The situation is, however, slightly more involved if the correlation functional already incorporates some $a b$ initio correlation, as the last family of density functional expressions known as Double-Hybrid (DH) models [19-21] or the approach known as Random-Phase-Approximation [22,23], which can capture (at least partially) this kind of interactions thanks to the non-locality of these correlation kernels. We will focus here on the former $\mathrm{DH}$ expressions, whose use has been widely extended in last years with remarkable accuracy [24]. We will try to accommodate (vide infra), as accurately as possible, the most used existing dispersion corrections on one hand, with parameter-free recent expressions on the other [25]. We will hereby study the coupling of the PBE-QIDH [26] and SOS1-PBE-QIDH [27] models with both pairwise and non-local dispersion corrections by: (i) deriving first the 
values of the functional-dependent parameters entering into the corrections, using well-established training sets (e.g. S130) for that; and (ii) applying these dispersion-corrected DH methods out of the training sets, to large supramolecular complexes (e.g. the L7 dataset), or to out-of-equilibrium regions (e.g. the S66x8 dataset), gaining thus insights into the performance of the methods and on the nature of the corrections merged with the $\mathrm{DH}$ expressions selected.

\section{Computational details}

A Double-Hybrid (DH) model corresponds usually to:

$$
E_{x c}^{\mathrm{DH}}[\rho]=\lambda_{x} E_{x}^{\mathrm{EXX}}[\phi]+\left(1-\lambda_{x}\right) E_{x}^{\mathrm{DFA}}[\rho]+\lambda_{c} E_{c}^{\mathrm{PT} 2}\left[\phi, \phi^{\prime}\right]+\left(1-\lambda_{c}\right) E_{c}^{\mathrm{DFA}}[\rho],
$$

with $\lambda_{x}\left(0 \leq \lambda_{x} \leq 1\right)$ and $\lambda_{c}\left(0 \leq \lambda_{c} \leq 1\right)$ scaling the different ingredients entering into its composition, as it is ordinarily done from the first developments of double-hybrid models [28]. Setting the PBE exchange and correlation individual functionals [29] for $E_{x}^{\mathrm{DFA}}[\rho]$ and $E_{c}^{\mathrm{DFA}}[\rho]$, respectively, together with values for parameters $\lambda_{x}$ and $\lambda_{c}$ derived from first-principles arguments, see Table 1, gives rise to the PBE-QIDH final expression. Note that QI stands for 'Quadratic Integrand', since a quadratic interpolation function was used to connect the non-interacting particle system with the real one, to find then the unknown exchange-correlation form upon integration. The SOS1-PBEQIDH variant scales differently the same- and opposite-spin contributions to the $E_{c}^{\mathrm{PT} 2}$ perturbative term, actually neglecting same-spin energies for com-

putational reasons [30]. We remind finally that $E_{x}^{\mathrm{EXX}}$ refers to the Exact-like EXchange (EXX) contribution, which is the common ingredient of former (hybrid) expressions. 
The built-in and very large Ahlrichs type quadruple- $\xi$ def2-QZVP basis set [31] was fixed for all the calculations reported here, which avoids the need of further corrections to eliminate the spurious basis set superposition error with smaller basis sets. We used the release 3.0.1 of the ORCA package [32] including the corresponding auxiliary (def2-QZVP/JK and def2-QZVP/C) basis sets for the more demanding calculations [33]. The numerical integration threshold were always strengthen with respect to the default values, to always be on the safer side for non-covalently bound complexes. The association or interaction energies for all the complexes were calculated as $\Delta E(A B)=E(A B)-E(A / / A B)-E(B / / A B)$, where $A B$ denotes the complex geometry.

\section{Results and discussion}

\subsection{Application of the PBE-QIDH and SOS1-PBE-QIDH models to the S130 dataset}

We first apply the uncorrected PBE-QIDH and SOS1-PBE-QIDH models to the S130 dataset of compounds [34,35], which includes as subsets: RG6 (rare gases dimers), ACONF (N-alkane conformers), ADIM6 (alkane dimers from ethane to n-heptane), S22 (non-covalently bound and H-bonded complexes), CYCONF (cysteine conformers), SCONF (sugar conformers), PCONF (small peptide conformers), and the S22+ extension (the S22 set at two stretched geometries for each of the complexes). This energy dataset has been extensively used before for assessing and/or parameterizing DH models, as it is also part of the more extended GMTKN30 benchmark database [36]. Table 2 shows the results for all the subsets, using the Mean Absolute De- 
viation (MAD) as the metrics for deviations with respect to benchmark references. From these results we remark: (i) The PBE-QIDH method consistently provides lower MADs for all the subsets of the S130 dataset, with MAD for individual subsets ranging between $0.2-1.2 \mathrm{kcal} / \mathrm{mol}$, compared with the B2-PLYP model, for which MAD for individual subsets are found to be between 0.2 and $2.8 \mathrm{kcal} / \mathrm{mol}$; (ii) A very low global MAD value of 0.42 $\mathrm{kcal} / \mathrm{mol}$ is obtained for the PBE-QIDH model; and (iii) the application of the SOS1-PBE-QIDH version gives a slightly higher MAD values for all subsets, leading nonetheless to a (still acceptably low) MAD of $0.61 \mathrm{kcal} / \mathrm{mol}$, which compares favourably for instance with the value of $0.63 \mathrm{kcal} / \mathrm{mol}$ provided by the spin-scaled DSD-PBEBP86 double-hybrid functional [37].

\subsection{Parameterization of the PBE-QIDH-D3(BJ) and SOS1-PBE-QIDH-D3(BJ) models}

We proceed next to optimize the parameters defining the D3(BJ) dispersion correction, as it was originally formulated [34,35]:

$$
E^{\mathrm{D} 3(\mathrm{BJ})}\left(R_{A B}\right)=-\sum_{n=6,8} s_{n} \sum_{B>A}^{\text {atom pairs }} \frac{C_{n}^{A B}}{R_{A B}^{n}+f_{n}\left(R_{A B}^{0}\right)},
$$

whose energy is added directly to that provided by Eq. (1), $E_{x c}=E_{x c}^{\mathrm{DH}}[\rho]+$ $E^{\mathrm{D} 3(\mathrm{BJ})}\left(R_{A B}\right)$, with $R_{A B}=\left|R_{A}-R_{B}\right|$ the internuclear distance between atoms $A$ and $B$. The dispersion correction is a function of: (i) a pair of functional-dependent parameters $\left(s_{n}\right)$ coupling efficiently both electronic and dispersion energies; (ii) atom-pair dependent $n$ th-order interatomic dispersion coefficients $C_{n}^{A B}$; and (iii) the damping function $f_{n}=\left(a_{1} R_{A B}^{0}+a_{2}\right)^{n}$, which allows to switch the energy from medium- to short-distances, with $R_{A B}^{0}=\sqrt{\frac{C_{8}^{A B}}{C_{6}^{A B}}}$, together with additional parameters $\left(a_{1}\right.$ and $\left.a_{2}\right)$ to be fit- 
ted $[38-40]$.

Thus, developing the first two terms of the above expression, the $s_{6}, s_{8}$, $a_{1}$, and $a_{2}$ parameters have to be determined, which is done here through the simplex algorithm on the S130 dataset, leading to the values gathered in Table 1 for both models and to the MAD values included in Table 2 . Interestingly, the D3(BJ) correction reduces the MAD to values as low as 0.18 and $0.15 \mathrm{kcal} / \mathrm{mol}$ for the PBE-QIDH-D3(BJ) and SOS1-PBE-QIDHD3(BJ) models, respectively, to be compared again with previous literature values of $0.13 \mathrm{kcal} / \mathrm{mol}$ for DSD-PBEBP86-D3(BJ) [37], $0.18 \mathrm{kcal} / \mathrm{mol}$ for PBE0-DH-D3(BJ) [41], or $0.21 \mathrm{kcal} / \mathrm{mol}$ at the B2-PLYP-D3(BJ) level [34]. Note that these deviations are lower in any case than those obtained using previous dispersion corrections for DH models [42, 43], i.e. the former -D2 variant, which were pioneering but are now possibly superseded. We also note in passing that the almost vanishing $a_{1}$ value obtained for SOS1-PBE-QIDHD3(BJ) agrees well with other spin-component-scaled DH functionals, like DSD-BLYP [44] and PWPB95 [45] both having a null $a_{1}$ value. We further explore this issue in line with a recent formulation of the D3(BJ) correction, dubbed as D3(CSO) [46], adapted to a fast algorithm for the computation of the corresponding gradient terms [47]. Setting thus $a_{1}=0$ in Eq. (2), but keeping the values exposed in Table 2 for the other parameters, negligibly change (actually by less than $0.05 \mathrm{kcal} / \mathrm{mol}$ ) the MAD for individual subsets of the S130 dataset, while simplifying the whole expression to:

$$
E^{\mathrm{D} 3(\mathrm{BJ})}\left(R_{A B}\right)=-\sum_{B>A}^{\text {atom pairs }}\left[s_{6} \frac{C_{6}^{A B}}{R_{A B}^{6}+a_{2}^{6}}+s_{8} \frac{C_{8}^{A B}}{R_{A B}^{8}+a_{2}^{8}}\right] \text {, }
$$

for the SOS1-PBE-QIDH-D3(BJ) case. Actually, this shows how this model needs a very simple damping function (a constant basically) for every pair of 
non-covalently interacting atoms.

\subsection{Parameterization of the PBE-QIDH-NL and SOS1- PBE-QIDH-NL models}

We also assess the viability of coupling the PBE-QIDH and SOS1-PBEQIDH models to a non-local correlation functional given by the expression [48]:

$$
E_{c}^{\mathrm{NL}}\left[\rho, \rho^{\prime}\right]=\int d \mathbf{r} \rho(\mathbf{r})\left[\frac{1}{2} \int d \mathbf{r}^{\prime} \Phi\left(\mathbf{r}, \mathbf{r}^{\prime}, b, C\right) \rho\left(\mathbf{r}^{\prime}\right)+\beta(b)\right],
$$

whose energy is again added to $E_{x c}^{\mathrm{DH}}[\rho]$, and thus $E_{x c}=E_{x c}^{\mathrm{DH}}[\rho]+E_{c}^{\mathrm{NL}}\left[\rho, \rho^{\prime}\right]$. Note that the function $\Phi\left(\mathbf{r}, \mathbf{r}^{\prime}\right)$ couples the total electronic densities at two different spatial points, $\rho(\mathbf{r})$ and $\rho\left(\mathbf{r}^{\prime}\right)$, keeping the correct asymptotic behavior $\left|\mathbf{r}-\mathbf{r}^{\prime}\right|^{-6}$, and that we resort to the modern Vydrov and van Voorhis (VV10) construction for the kernel [49]. Note that while the latter D3(BJ) correction is fully semiempirical (tabulation of all the dispersion coefficients for each atom) this formalism limits empiricism and can be viewed as part of the density-dependent dispersion corrections [50-54].

In this case, it has been repeatedly shown how an efficient coupling between both expressions, $E_{x c}[\rho]$ and $E_{c}\left[\rho, \rho^{\prime}\right]$, is done through the densityindependent term $\beta=\frac{1}{32}\left(\frac{3}{b^{2}}\right)^{3 / 4}$ and more specifically by finding the optimal value of $b$ independently of the type (semi-local, hybrid or double-hybrid) of functional [55-58]. We thus adopt the same strategy here and optimize $b$ against reference values of the S22 dataset [59,60], a pioneer and widely used database [61] which would also allow us to compare with previous results for other DH models. We find $b=14.2$ (PBE-QIDH-NL) and $b=8.1$ (SOS1PBE-QIDH-NL) leading to MAD values of only 0.30 and $0.24 \mathrm{kcal} / \mathrm{mol}$, re- 
spectively, and thus very competitive with respect to previous calculations (ie. $0.21 \mathrm{kcal} / \mathrm{mol}$ for B2PLYP-NL or 0.46 for PBE0-DH-NL) [57]. Once these values were obtained, we also tested small variations (by $10 \%$ ) of the other parameter (dubbed as $C=0.0093$ ) entering into the kernel, finding a completely negligible impact on the MAD values previously declared, in agreement with other studies [62].

We further decompose the S22 complexes into a pair of subsets according to the main underlying non-covalent force (electrostatic or dispersion) between the monomers, following the recommendations given in Refs. [63, 64], to see that the MAD for the dispersion-dominated subset is less than 0.1 $\mathrm{kcal} / \mathrm{mol}$ for both PBE-QIDH-NL and SOS1-PBE-QIDH-NL models, but that it ranges between $0.4-0.6 \mathrm{kcal} / \mathrm{mol}$ for $\mathrm{H}$-bonded complexes, and thus still slightly overbinding those complexes. The optimal value of the $b$ parameter does not significantly change (ie. by less than $5 \%$ ) if we optimize it separately for each of these subsets, thus indicating the compromise of the $E_{x c}^{\mathrm{DH}}[\rho]+E_{c}^{\mathrm{NL}}\left[\rho, \rho^{\prime}\right]$ coupling for all kind of non-covalent interactions. We also believe that double-counting effects, arising from the coupling of a DH functional for short- and intermediate-range interactions to a functional suited for long-range interactions between weakly overlapped densities, are minimized here due to the flexibility of the $E_{c}^{\mathrm{NL}}$ form to adjust the short-range damping through the $b$ parameter, as it was also recently demonstrated for other density functionals combinations [65] and/or for compressed geometries out of equilibrium (the S66x8 database) of non-covalently bound systems [66]. 


\subsection{Application to the L7 dataset}

We also assess the performance of all the models derived here for the L7 dataset of large supramolecular systems [67], which comprises supramolecular complexes mostly dispersion-dominated (aliphatic-aliphatic and $\pi-\pi$ interactions) and with a large molecular size reaching up to 112 atoms (see Figure 1). These systems are known to push theoretical methods to their accuracy limit [68], as it is also evidenced by current difficulties to obtain benchmark results at the Complete Basis Set (CBS) limit and with a method as accurate as CCSD(T). This issue was recently solved by resorting to the domain-based pair natural orbital DLPNO-CCSD(T) approach at the CBS limit, offering nearly $\operatorname{CCSD}(\mathrm{T})$ accuracy at a reduced computational cost [69], and thus avoiding the use of previously published QCISD(T)/CBS values [67].

The PBE-QIDH method systematically underbinds all the complexes, as it is clearly seen from the Mean Signed Deviation (MSD) values gathered in Table 3, although it still provides a decent MAD value of $6.4 \mathrm{kcal} / \mathrm{mol}$ which prompts for the use of a dispersion correction. Note that previous results from literature provided a MAD of $11.8 \mathrm{kcal} / \mathrm{mol}$ for the B2-PLYP method [68], with the def2-TZVP basis set but counterpoise corrected, and thus much higher than that obtained here. Interestingly, the use of the D3(BJ) correction leads now to a very low MSD (MAD) of only 1.3 (1.9) $\mathrm{kcal} / \mathrm{mol}$, and very close to that provided by other dispersion-corrected $\mathrm{DH}$ methods (e.g. B2PLYP-D3 drops a MAD of $1.5 \mathrm{kcal} / \mathrm{mol}$, see Ref. [68]). We underline that the three-body and geometry-based, Axilrod-Teller-Muto expression [70], and thus without needing any further parameterization, was also used to correct the D3(BJ) energies, contributing with repulsive values ranging from 0.2 (the stacked guanine trimer) to 1.7 (a stacked circum- 
coronene bound to a guanine-cytosine dimer) kcal/mol, correlating thus with the size of the systems and concomitantly showing the importance of manybody effects for large supramolecular systems. The use of the NL correction decreases the MAD value up to $1.4 \mathrm{kcal} / \mathrm{mol}$, to be compared again with the $1.2 \mathrm{kcal} / \mathrm{mol}$ provided by B2PLYP-NL [68], and leads to a negligibly low MSD less than $-0.1 \mathrm{kcal} / \mathrm{mol}$.

On the other hand, the performance of the SOS1-PBE-QIDH variant initially deviates from that of pristine PBE-QIDH, with a larger underbinding and even (wrongly) predicting an unbound stacked guanine trimer. The D3(BJ) correction helps again to largely reduced the deviation, but still with some margin for further improvements. Finally, the coupling with the nonlocal correlation functional gives a MSD (MAD) as low as - $0.2(1.5) \mathrm{kcal} / \mathrm{mol}$, and again in the range of "chemical accuracy" (ie. a deviation of $1-2 \mathrm{kcal} / \mathrm{mol}$ with respect to reference results) for non-covalently bound systems.

\subsection{Application to the $\mathrm{S} 66 \times 8$ dataset}

The S66x8 dataset of non-covalent interactions [71,72] includes each dimer of the original S66 set at eight (x8) intermolecular separations, that is, at $0.90 R_{e}, 0.95 R_{e}, 1.00 R_{e}, 1.05 R_{e}, 1.10 R_{e}, 1.25 R_{e}, 1.50 R_{e}$, and $2.00 R_{e}$, where $R_{e}$ is the equilibrium distance, and thus encompassing repulsive $\left(R<R_{e}\right)$ and attractive $\left(R>R_{e}\right)$ regions. The application of our dispersion-corrected methods to this set of energies would allow a clear understanding of the strength and range of validity of the dispersion corrections employed. Note that we use as reference values the benchmark results recently obtained at the $\operatorname{CCSD}\left(-\mathrm{F}_{12}\right)(\mathrm{T})$ level [66], which might differ up to $0.3 \mathrm{kcal} / \mathrm{mol}$ with 
respect to former $\operatorname{CCSD}(\mathrm{T}) / \mathrm{CBS}$ reference energies.

Figure 2 shows the evolution of the MAD at all intermolecular distances, and for all the methods considered, that is PBE-QIDH, PBE-QIDH-D3(BJ), PBE-QIDH-NL, SOS1-PBE-QIDH, SOS1-PBE-QIDH-D3(BJ), and SOS1PBE-QIDH-NL. As a general trend, it can be easily seen how the MAD is always greatly reduced for all models when going from repulsive to attractive regions, including a dispersion correction or not. Looking at the behavior of the pristine (i.e. dispersion-uncorrected) PBE-QIDH and SOS1-PBE-QIDH methods, and considering as a metric the global MAD obtained averaging the energy deviations for all distances, values of 0.66 and $1.30 \mathrm{kcal} / \mathrm{mol}$ are obtained, respectively. The figure also serves to confirm once again the advantage of using $\mathrm{DH}$ functionals for intermediate (e.g. between $1.00 R_{e}$ and $1.50 R_{e}$ ) regions, with a pronounced error decrease as a function of the marked slope found for that range of distances.

Particularly accurate is the performance of the PBE-QIDH-D3(BJ) and PBE-QIDH-NL methods along the whole set of points, for which a MAD lower than $0.4 \mathrm{kcal} / \mathrm{mol}$ is found for all of the distances and reaching deviations as low as $0.1 \mathrm{kcal} / \mathrm{mol}$ at the longest (i.e. $1.25 R_{e}, 1.50 R_{e}$, and $2.00 R_{e}$ ) ones considered. That gives a global MAD of only $0.22 \mathrm{kcal} / \mathrm{mol}$ for both models, in agreement with other accurate methods like B2GP-PLYP-D3(BJ) or DSD-PBEP86-D3(BJ) [66]. By perusing now the corresponding values for SOS1-PBE-QIDH-D3(BJ) and SOS1-PBE-QIDH-NL variants, we now obtain MAD of 0.40 and $0.18 \mathrm{kcal} / \mathrm{mol}$, respectively. Interestingly, the behavior of the former method deviates significantly in the region $0.90 R_{e}-1.10 R_{e}$, probably due to the low value of the $a_{1}$ parameter found. 


\section{Conclusions}

We demonstrate here how the PBE-QIDH and SOS1-PBE-QIDH doublehybrid density functionals already describe semi-quantitatively the energetics for the association of weakly bound supramolecular complexes at all intermediate- and long-range distances. Furthermore, we would like to highlight how the D3(BJ) and NL dispersion corrections can be successfully incorporated into the PBE-QIDH model, providing averaged energy deviations below 1 (between 1-2) kcal/mol for small (large) complexes, and thus very close to those obtained by the best performing parameterized DH expressions. These low deviations are also kept for stretched and elongated distances, as it is found upon computation of the S66x8 dataset of molecular complexes at distances ranging from $0.90 R_{e}$ to $2.00 R_{e}$. The non-local van der Waals correction VV10 seems to be specially suited for that, once the short-range attenuation parameter $b$ is carefully optimized. We also extend the fitting of the dispersion corrections to the spin-scaled SOS1-PBE-QIDH variant, which however suffered from a larger mean absolute deviation for the L7 and S66x8 datasets with the D3(BJ) correction with respect to the non-local one. Overall, it seems that these corrected methods can be safely recommended for energy differences driven by non-covalent effects, merging all (short-, intermediate-, and long-range) regimes into a compact, and easily accessible (ie. available in most codes) density expression, although it might slightly overbind the association energies of H-bonded dimers. We will finally like to stress here how it is possible to obtain very good estimates of non-covalent energies with parameter-free double-hybrid density functionals, once they are efficiently combined with state-of-the-art dispersion corrections. 


\section{Acknowledgements}

JCSG and AJPJ thank the "Ministerio de Economía y Competitividad" of Spain and the "European Regional Development Fund" through project CTQ2014-55073-P. JCSG would also like to thank J. Aragó and J. Calbo (University of Valencia) for providing the MUE for the L7 dataset employing the B2-PLYP method, and for inspiring discussions.

\section{References}

[1] Trouillas, P. ; Sancho-García, J. C.; De Freitas, V.; Gierschner, J.; Otyepka, M.; Dangles, O. Chem. Rev. 2016, 116, 4937.

[2] Lazar, P.; Karlický, F.; Jurečka, P.; Kocman, M.; Otyepková, E.; Šafá řová, K.; Otyepka, M. J. Am. Chem. Soc. 2013, 135, 6372.

[3] Pykal, M.; Jurečka, P.; Karlický, F.; Otyepka, M. Phys. Chem. Chem. Phys. 2016, 18, 6351.

[4] Otyepková E.; Lazar P.; Cepe K.; Tomanec O.; Otyepka M. Appl. Mat. Today 2016, 5, 142.

[5] Ulbrich, K.; Holá, K.; Šubr, V.; Bakandritsos, A.; Tuček, J.; Zbořil, R. Chem. Rev. 2016, 116, 5338.

[6] Biedermann, F.; Schneider, H.-J. Chem. Rev. 2016, 116, 5216.

[7] Č́rny, J.; Hobza, P. Phys. Chem. Chem. Phys. 2007, 9, 5291.

[8] Grimme, S.; Antony, J.; Schwabe, T.; Mück-Lichtenfeld, C. Org. Biomol. Chem. 2007, 5, 741. 
[9] Johnson, E. R.; Mackie, I. D.; DiLabio, G. A. J. Phys. Org. Chem. 2009, 22, 1099.

[10] Foster, M. E.; Sohlberg, K. Phys. Chem. Chem. Phys. 2010, 12, 307.

[11] Riley, K. E.; Pitoňák, M.; Jurečka, P.; Hobza, P. Chem. Rev. 2010, 110, 5023.

[12] Hobza, P. Annu. Rep. Prog. Chem., Sect. C 2011, 10\%, 148.

[13] Grimme, S. WIREs Comput. Mol. Sci. 2011, 1, 211.

[14] Klimeš, J.; Michaelides, A. J. Chem. Phys. 2012, 137, 120901.

[15] Ehrlich, S.; Moellmann, H.; Grimme, S. Acc. Chem. Res. 2013, 45, 916.

[16] DiStasio Jr., R. A.; Gobre, V. V.; Tkatchenko, A. J. Phys.: Condens. Matter 2014, 26, 213202.

[17] Corminboeuf, C. Acc. Chem. Res. 2014, 47, 3217.

[18] Grimme, S.; Hansen, A.; Brandenburgand, J. G.; Bannwarth, C. Chem. Rev. 2016, 116, 5105.

[19] Sancho-García, J. C.; Adamo, C. Phys. Chem. Chem. Phys. 2013, 15, 14581.

[20] Goerigk, L.; Grimme, S. WIREs Comput. Mol. Sci. 2014, 4, 576.

[21] Su, N. Q.; Xu, X. WIREs Comput. Mol. Sci. 2016, 6, 721.

[22] Wuming, Zhu.; Toulouse, J.; Savin, A.; Ángyán, J. G. J. Chem. Phys. 2010, 132, 244108.

[23] Ren, X., Rinke, P., Joas, C., Scheffler, M. J. Mater. Sci. 2012, 47, 7447. 
[24] Bousquet, D.; Brémond, E.; Sancho-García, J. C.; Ciofini, I.; Adamo, C. J. Chem. Theory Comput. 2013, 9, 3444.

[25] Brémond, E.; Ciofini, I.; Sancho-García, J. C.; Adamo, C. Acc. Chem. Res. 2016, 49, 1503.

[26] Brémond, E.; Sancho-García, J. C.; Pérez-Jiménez, A. J.; Adamo, C. J. Chem. Phys. 2014, 141, 031101.

[27] Brémond, E.; Savarese, M.; Sancho-García, J. C.; Pérez-Jiménez, A. J.; Adamo, C. J. Chem. Phys. 2016, 144, 124104.

[28] Grimme, S. J. Chem. Phys. 2006, 124, 034108.

[29] Perdew, J. P.; Burke, K.; Ernzerhof, M. Phys. Rev. Lett. 1996, 77, 3865.

[30] Jung, Y.; Lochan, R. C.; Dutoi, A. D.; Head-Gordon, M. J. Chem. Phys. 2004, 121, 9793.

[31] Weigend T.; Ahlrichs R. Phys. Chem. Chem. Phys. 2005, 7, 3297.

[32] Neese, F. WIREs Comput. Mol. Sci. 2012, 2, 73.

[33] Neese, F.; Wennmohs, F.; Hansen, A.; Becker, U. Chem. Phys. 2009, $356,98$.

[34] Grimme, S.; Antony, J.; Ehrlich, S.; Krieg, H. J. Chem. Phys. 2010, 132, 154104.

[35] Grimme, S.; Ehrlich, S.; Goerigk, L. J. Comput. Chem. 2011, 32, 1456.

[36] Goerigk, L.; Grimme, S. Phys. Chem. Chem. Phys. 2011, 13, 6670.

[37] Kozuch, S.; Martin, Jan M. L. Phys. Chem. Chem. Phys. 2011, 13, 20104. 
[38] Becke, A. D.; Johnson, E. R. J. Chem. Phys. 2005, 122, 154101.

[39] Johnson, E. R.; Becke, A. D. J. Chem. Phys. 2005, 123, 024101.

[40] Johnson, E. R.; Becke, A. D. J. Chem. Phys. 2006, 124, 174104.

[41] Bousquet, D.; Brémond, E.; Sancho-García, J. C.; Ciofini, I.; Adamo, C. Theor. Chem. Acc. 2015, 134, 1602.

[42] Schwabe, T.; Grimme, S. Phys. Chem. Chem. Phys. 2007, 9, 3397.

[43] Sancho-García, J. C. Chem. Phys. Lett. 2012, 535, 136.

[44] Kozuch, S.; Gruzman, D.; Martin, Jan M. L. J. Phys. Chem. C 2010, 114, 20801.

[45] Goerigk, L.; Grimme, S. J. Chem. Theory Comput. 2011, 7, 291.

[46] Schrodër, H.; Creon, A.; Schwabe, T. J. Chem. Theory Comput. 2015, 11,3163 .

[47] Schrodër, H.; Hühnert, J. Schwabe, T. J. Chem. Phys. 2017, 146, 044115 .

[48] Calbo, J.; Ortí, E.; Sancho-García, J. C.; Aragó, J. Ann. Rep. Comput. Chem. 2015, 11, 37.

[49] Vydrov, O. A.; Van Voorhis, T. J. Chem. Phys. 2010, 133, 244103.

[50] Becke, A. D.; Johnson, E. R. J. Chem. Phys. 2007, 127, 154108.

[51] Tkatchenko, A.; Scheffler, M. Phys. Rev. Lett. 2009, 102, 073005.

[52] Sato, T.; Nakai, H. J. Chem. Phys. 2010, 133, 194101. 
[53] Steinmann, S. N.; Corminboeuf, C. J. Chem. Theory Comput. 2011, 7, 3567.

[54] Brémond, E.; Golubev, N.; Steinmann, S. N.; Corminboeuf, C. J. Chem. Phys. 2014, 140, 18A516.

[55] Hujo, W.; Grimme, S. J. Chem. Theory Comput. 2011, 7, 3866.

[56] Hujo, W.; Grimme, S. J. Chem. Theory Comput. 2013, 9, 308.

[57] Aragó, J.; Ortí, E.; Sancho-García, J. C. J. Chem. Theory Comput. 2013, 9, 3437.

[58] Kesharwani, M. J.; Karton, A.; Martin, Jan M. L. J. Chem. Theory Comput. 2016, 12, 444.

[59] Tatakani, T.; Hohenstein, E. G.; Malagoli, M.; Marshall, M. S.; Sherrill, C. D. J. Chem. Phys. 2010, 132, 144104.

[60] Marshall, M. S.; Burns, L. A.; Sherrill, C. D. J. Chem. Phys. 2011, 135, 194102

[61] Jurečka, P.; Sponer, J.; Černy, J.; Hobza, P. Phys. Chem. Chem. Phys. 2006, 8, 1985.

[62] Mardirossian, N; Head-Gordon, M. J. Chem. Phys. 2016, 144, 214110.

[63] Gráfová, L.; Pitoňák, M.; Rezáč, J.; Hobza, P. J. Chem. Theory Comput. 2010, 6, 2365.

[64] Flick, J. C.; Kosenkov, D.; Hohenstein, E. G.; Sherrill, C. D.; Slipchenko, L. V. J. Chem. Theory Comput. 2012, 8, 2835. 
[65] Peng, H.; Yang, Z.-H.; Perdew, J. P.; Sun, J. Phys. Rev. X 2016, 6, 041005.

[66] Brauer, B.; Kesharwani, M. K.; Kozuch, S.; Martin, Jan M. L. Phys. Chem. Chem. Phys. 2016, 18, 20905.

[67] Sedlak, R.; Janowski, T.; Pitok, M.; Rez, J.; Pulay, P.; Hobza, P. J. Chem. Theory Comput. 2013, 9, 3364.

[68] Calbo, J.; Ortí, E.; Sancho-García, J. C.; Aragó, J. J. Chem. Theory Comput. 2015, 11, 932.

[69] Riplinger, C.; Sandhoefer, B.; Hansen, A.; Neese, F. J. Chem. Phys. 2013, 139, 134101.

[70] von Lilienfeld, O. A.; Tkatchenko, A. J. Chem. Phys. 2010, 132, 234109.

[71] Rezav́ c, J.; Riley, K. E.; Hobza, P. J. Chem. Theory Comput. 2011, 7, 2427.

[72] Rezav́ c, J.; Riley, K. E.; Hobza, P. J. Chem. Theory Comput. 2011, 7, 3466. 
- Table 1. Summary of the $\lambda_{x}$ and $\lambda_{c}$ values entering into the QIDH functionals selected, as well as the list of empirical coefficients for the dispersion corrections used.

- Table 2. Mean Absolute Deviations (in kcal/mol) for interaction energies of the subsets of the S130 dataset.

- Table 3. Mean Signed and Absolute Deviations (in kcal/mol) for interaction energies of the L7 dataset, with respect to the DLPNO$\operatorname{CCSD}(\mathrm{T})$ reference energies. 
Table 1:

\begin{tabular}{lllllllll}
\hline Method & $\lambda_{x}$ & $\lambda_{c}$ & $\left(1-\lambda_{c}\right)$ & $s_{6}$ & $a_{1}$ & $s_{8}$ & $a_{2}$ & $b$ \\
\hline PBE-QIDH & $3^{-1 / 3}$ & $1 / 3$ & $2 / 3$ & 0.610 & 0.114 & 0.566 & 7.538 & 14.2 \\
SOS1-PBE-QIDH & $3^{-1 / 3}$ & $4 / 9$ & $2 / 3^{\mathrm{a}}$ & 0.750 & 0.002 & 0.200 & 6.794 & 8.1 \\
\hline
\end{tabular}

${ }^{\text {a }}$ Note the unexpected scaling due to the spin-opposite-scaling construction of the model, see Ref. [27] for further details. 
Table 2:

\begin{tabular}{llllll}
\hline & weight $^{\mathrm{a}}$ & PBE-QIDH & PBE-QIDH-D3(BJ) & SOS1-PBE-QIDH & SOS1-PBE-QIDH-D3(BJ) \\
\hline ACONF & 15 & 0.184 & 0.075 & 0.341 & 0.051 \\
ADIM6 & 6 & 1.459 & 0.370 & 2.186 & 0.324 \\
CYCONF & 10 & 0.498 & 0.552 & 0.254 & 0.359 \\
$\quad$ & 22 & 0.776 & 0.586 & 1.195 & 0.411 \\
S22 & 44 & 0.510 & 0.200 & 0.763 & 0.176 \\
S22+ & 17 & 0.132 & 0.099 & 0.522 & 0.310 \\
SCONF & 10 & 1.249 & 0.619 & 1.628 & 0.421 \\
PCONF & 10 & 0.035 & 0.363 & 0.027 \\
RG6 & $6 \times 20^{\mathrm{b}}$ & 0.273 & 0.18 & 0.61 & 0.15 \\
\hline
\end{tabular}

a The weight indicates the number of reference energies included.

b The RG6 reference energies are weighted by a factor of 20, as it is normally done when employing the S130 dataset for fitting purposes. 
Table 3:

\begin{tabular}{|c|c|c|}
\hline Method & MSD & MAD \\
\hline PBE-QIDH & 6.45 & 6.45 \\
\hline PBE-QIDH-D3(BJ) ${ }^{\mathrm{a}}$ & 1.29 & 1.89 \\
\hline PBE-QIDH-NL & -0.06 & 1.40 \\
\hline SOS1-PBE-QIDH & 12.4 & 12.4 \\
\hline SOS1-PBE-QIDH-D3(BJ) ${ }^{\mathrm{a}}$ & 4.00 & 4.30 \\
\hline SOS1-PBE-QIDH-NL & -0.16 & 1.48 \\
\hline
\end{tabular}


- Figure 1. Chemical structures of the complexes forming the L7 dataset. The hydrogen atoms and corresponding $\mathrm{C}-\mathrm{H}$ bonds have been omitted for clarity. Color code for atoms: C (grey), $\mathrm{H}$ (white), $\mathrm{N}$ (blue), O (red).

- Figure 2. Evolution of the MAD for different methods, as a function of the intermolecular distance, for the $\mathrm{S} 66 \mathrm{x} 8$ dataset of compounds. 


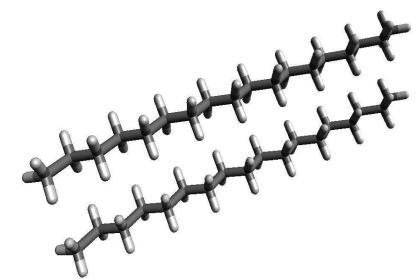

Octadecane dimer

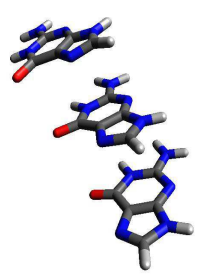

G trimer

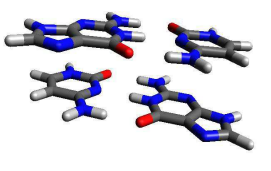

GC base pair stack

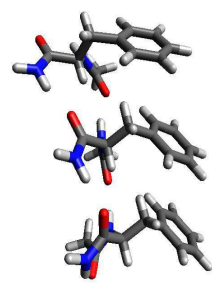

Phenylalanine residues trimer
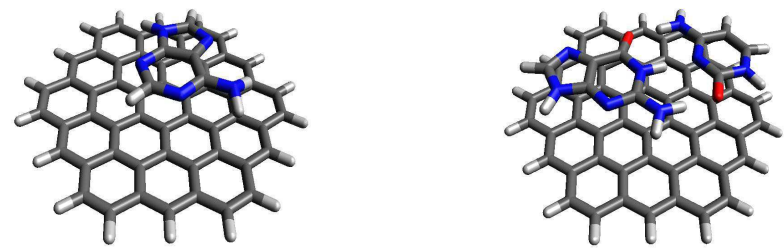

Circumcoronene \& GC base pair

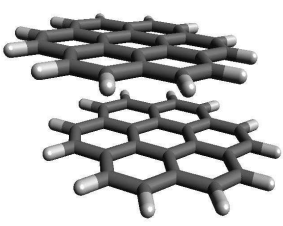

Coronene dimer

Figure 1. 


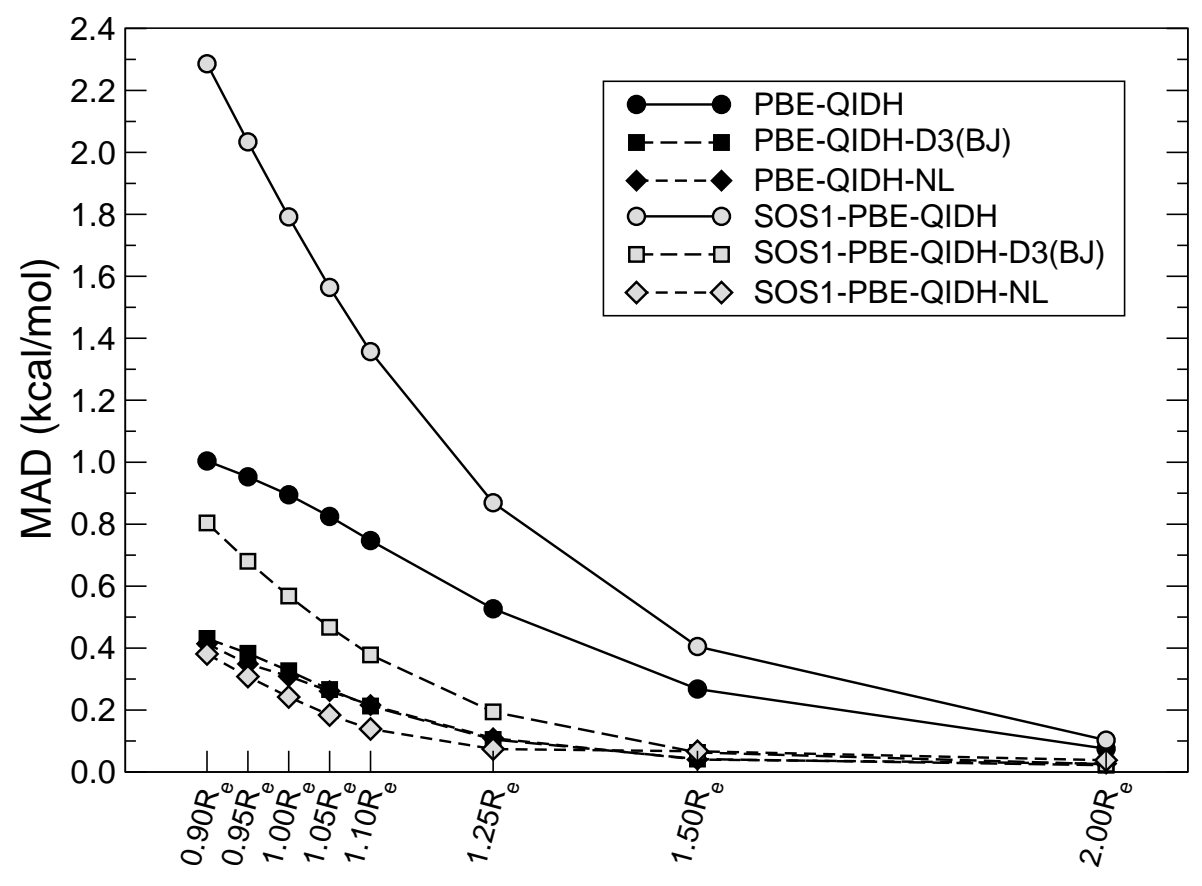

Figure 2. 